JAIME DÍAZ PACHECOa

ABEL LÓPEZ DÍEZ

AMALIA YANES LUQUE

PEDRO DORTA ANTEQUERA ${ }^{\mathrm{d}}$

PABLO MÁYER SUÁREZ

\title{
PROPUESTA METODOLÓGICA PARA ESTIMAR LA VULNERABILIDAD LOCAL POR INUNDACIÓN EN ÁREAS TURÍSTICAS COSTERAS DE CLIMA ÁRIDO: APLICACIÓN AL LITORAL DE ARONA Y ADEJE (SO DE TENERIFE)
}

\begin{abstract}
RESUMEN
En este trabajo se propone una metodología cualitativa para el cálculo de un índice de vulnerabilidad por inundación. Partiendo de información disponible en muchas administraciones locales, esta se aplica en el litoral de Arona y Adeje, áreas turísticas en el suroeste de Tenerife que cuentan con una oferta alojativa superior a las 90.000 plazas, como consta en el Instituto de Estadística del Gobierno de Canarias. De la labor desarrollada a nivel de parcela catastral resulta un índice de vulnerabilidad que oscila entre un valor mínimo de 0 y un máximo 1 , así como su diferenciación espacial según la antigüedad de las urbanizaciones turísticas. El empleo del índice que se formula permite evaluar a escala local y con gran precisión la vulnerabilidad de espacios turísticos.
\end{abstract}

a Universidad de La Laguna, Departamento de Geografía e Historia y Cátedra Universitaria "Reducción del riesgo de desastres y ciudades resilientes”. jdiazpac@ull.es.

b Universidad de La Laguna, Departamento de Geografía e Historia y Cátedra Universitaria "Reducción del riesgo de desastres y ciudades resilientes”. alopezd@ull.es.

c Universidad de La Laguna, Departamento de Geografía e Historia. ayanes@ull.es.

d Universidad de La Laguna, Departamento de Geografía e Historia y Cátedra Universitaria "Reducción del riesgo de desastres y ciudades resilientes". pdorta@ull.es.

e Universidad de Las Palmas de Gran Canaria, Grupo de Geografía Física y Medio Ambiente, Instituto de Oceanografía y Cambio Global (IOCAG). pablo.mayer@ulpgc.es.

Fecha de recepción: 27/1/20. Fecha de aceptación: 22/4/20. 
Palabras clave: costa; desastres; parcela catastral; riesgo de inundación; Tenerife; vulnerabilidad.

\title{
METHODOLOGICAL PROPOSAL TO ESTIMATE LOCAL VULNERABILITY TO FLOODING IN ARID CLIMATE COASTAL TOURIST AREAS: APPLICATION TO THE COASTLINE OF ARONA AND ADEJE (SW OF TENERIFE)
}

\begin{abstract}
This paper proposes a qualitative methodology for calculating a flood vulnerability index. Based on information available in many local administrations, it is applied in the Arona and Adeje coast, tourist areas in the southwest of Tenerife that have a lodging offer of over 90.000 places annually (ISTAC, 2018). From the work carried out at the cadastral plot level, a vulnerability index is extracted that ranges between a minimum value of 0 and a maximum of 1 , as well as its spatial differentiation according to the age of the tourist urbanizations. This index is a good method for evaluations of urban spaces vulnerability
\end{abstract}

KeYwords: cadastral plot; coast; disasters; flood risk; Tenerife; vulnerability.

\section{LA VUlNERABILIDAd LOCAL COMO OBJETIVO DE ANÁLISIS EN ESPACIOS CON RIESGO DE INUNDACIÓN}

La guía para la adaptación y la elaboración de planes locales de adaptación al Cambio Climático del Ministerio de Agricultura, Pesca y Alimentación Gobierno de España (MAPAMA, 2015) define la vulnerabilidad del territorio según su propensión a ser afectado por una amenaza. En iguales términos se expresan los informes para la gestión del riesgo de eventos extremos y desastres del Panel Intergubernamental sobre el Cambio Climático (IPCC, 2014). No obstante, en sus descripciones la vulnerabilidad también se contempla como un conjunto de caracteres y condicionantes fruto de la realidad social, cultural, histórica, medioambiental, política y económica en la que la sociedad desarrolla sus actividades (Cardona, 2012). Unos y otros podrían configurar los ejes básicos de diversos indicadores, que posibiliten medir y/o evaluar de forma adecuada la vulnerabilidad de un territorio o de una comunidad ante una amenaza determinada. Además, en el contexto del Cambio Climático aquella se construye, así mismo, en función de la exposición, la sensibilidad y la capacidad de adaptación de todo grupo humano (McCarthy et al., 2004; Füssel and Klein, 2006; G. O’Brien et al., 2008). En este sentido, la exposición aparece como factor tácito y objetivo al que la vulnerabilidad se vincula casi en su práctica totalidad (Burton et al., 1993). Ello es evidente sobre todo cuando se trata de riesgos específicos, como los que entrañan las inundaciones dadas sus repercusiones en la población y en el territorio.

La presencia y la integración de las variables a partir de las que sopesar la vulnerabilidad y precisar el grado de detalle de su evaluación están subordinadas, en lo esencial, a la escala de análisis a emplear y a la disponibilidad de datos al respecto: en el primer caso, porque puede ser continental, nacional, regional o local (Ruiz Pérez, 2011, p. 47); en el segundo, porque el volumen óptimo de registros no es siempre alcanzable. La combinación de estos dos aspectos introduce un margen de heterogeneidad 
y diferenciación espacial apreciable en su análisis. De ahí que sea de gran utilidad, desde una óptica geográfica, el tratamiento y la representación cartográfica de la información a escala local para evaluar, planificar y mitigar el riesgo.

Partiendo de estas consideraciones, la finalidad del presente trabajo es realizar una aproximación a un método de valoración y zonificación, a escala de parcela catastral, de la vulnerabilidad frente al riesgo de inundación. Para ello, la labor a efectuar se orienta, de un lado, al establecimiento de indicadores cualitativos de amenaza-exposición y vulnerabilidad, previa clasificación de los elementos en riesgo; y, de otro, a comprobar su viabilidad mediante la aplicación en espacios sensibles a las avenidas, de los que son exponentes claros los municipios de Arona y Adeje en el suroeste de Tenerife. Su elección deriva de la concurrencia de diversos factores medioambientales y socioeconómicos, que propician, al igual que en otras regiones del globo, situaciones de peligro. Entre ellos cabe destacar el ser áreas costeras con alto grado de ocupación, regímenes pluviométricos áridos con escorrentías esporádicas pero violentas y una actividad económica basada en el turismo, al que se debe una urbanización rápida y falta, por lo común, de planificación adaptada al medio.

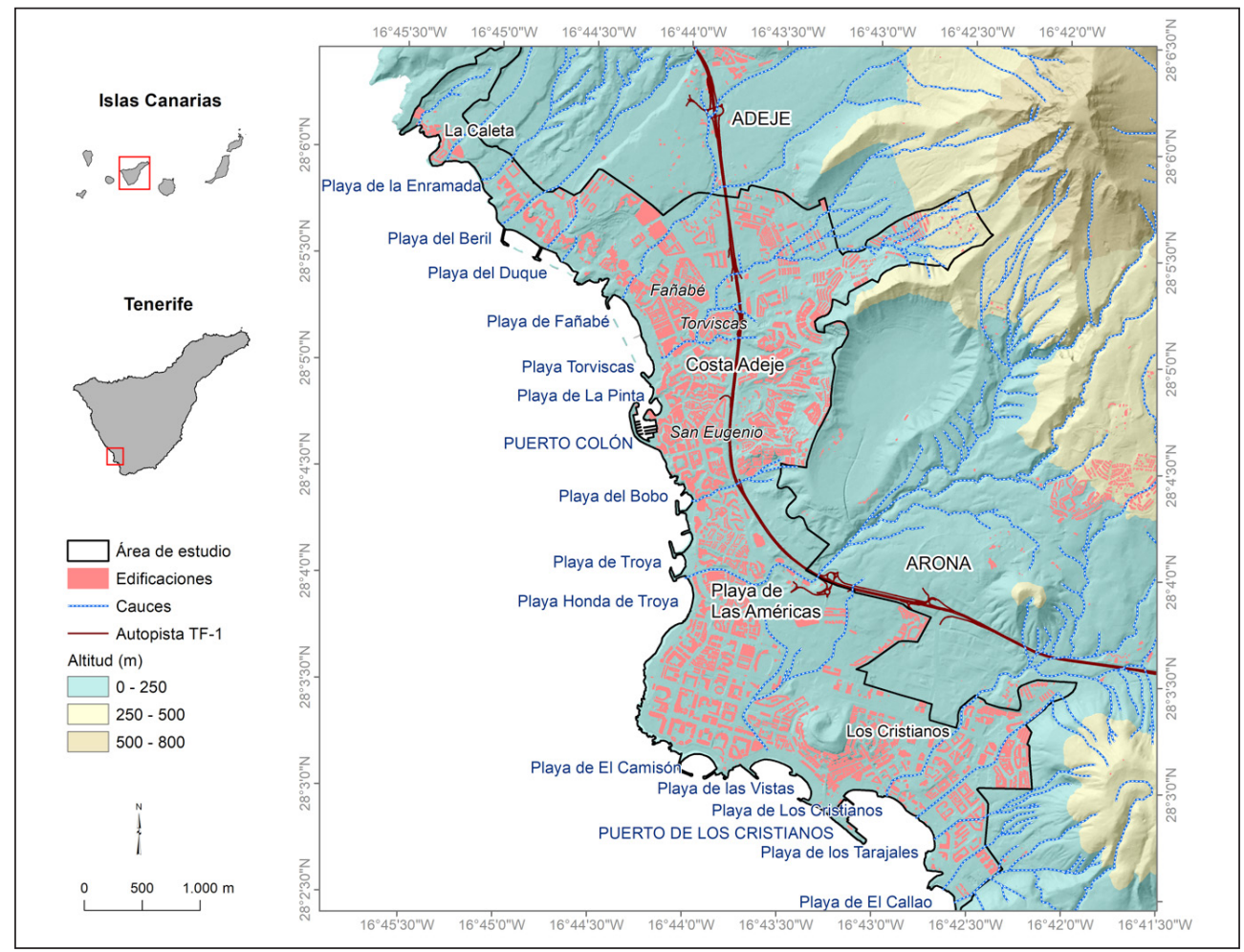

Figura 1. Localización y delimitación del área de estudio. Costa de Arona y Adeje (SO de Tenerife). Fuente: Grafcan e IGN (2019). 


\section{El ÁReA De Estudio en Un CONTEXTO DE RIESGO}

El ámbito objeto de análisis es una franja de $16,2 \mathrm{~km}^{2}$, que se extiende a lo largo de los 19,1 km del litoral de Arona y Adeje y los 100 metros de altitud. Se trata de un espacio de vocación turística manifiesta, considerando su pertenencia a los dos municipios que albergan no solo los centros neurálgicos del turismo tinerfeño, sino también de los más importantes de España (figura 1). Lo evidencia el volumen de su infraestructura y una oferta alojativa en 2018 de 91.266 plazas con una ocupación media anual que ronda el 73\% (ISTAC; Díaz-Pacheco et al., 2019); a esto se suma el carecer de la estacionalidad propia de los destinos turísticos mediterráneos.

La transformación del modelado y dinámica litoral por acción antrópica es rasgo distintivo del área que se estudia, fruto de la acomodación impuesta por una industria demandante, como ocurre en todo ámbito turístico, de espacio adecuado para satisfacer las exigencias de recreación y ocio junto al mar de un número de usuarios que no deja de aumentar (Roig et al., 2011). Lo que en principio era una costa jalonada por acantilados sobre coladas y depósitos sedimentarios, plataformas de abrasión y playas de cantos en terrazas y desembocadura de barrancos, ha sido sustituida por complejos residenciales, paseos marítimos, terrazas turísticas, obras portuarias y playas regeneradas de modo artificial provistas de diques de contención de arenas. La costa de Arona y Adeje es en la actualidad un continuo edificado sin espacios vacíos que completar, en el que se suceden casi sin interrupción playas urbanas, como Los Tarajales, Los Cristianos y Las Vistas, en Arona, y El Bobo, La Pinta, Torviscas, Fañabé, El Duque, en Adeje entre otras: playas definidas no tanto por la accesibilidad y dotación de servicios de baño, como por la fragilidad y vulnerabilidad ocasionadas por la alteración de su equilibrio natural.

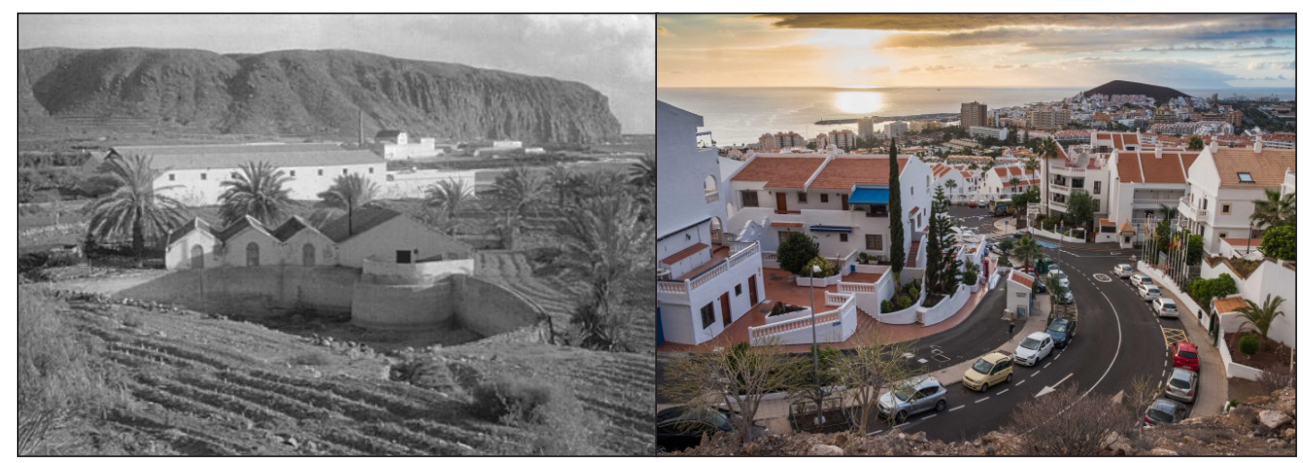

Figura 2: Cambio de usos del suelo en la costa de Los Cristianos, en el SE de Arona (SO de Tenerife): del secano agrícola en la primera mitad del siglo XX al desarrollo turístico actual. Fuente: Anónima (imagen izquierda) y Autores (imagen derecha). 
La saturación demográfica y urbanística de este litoral entronca con los cambios de uso del suelo registrados en Canarias desde la década de 1960 por la adopción de un modelo de desarrollo turístico-urbanizador, que deriva en una litoralización inusitada del territorio insular (Pérez-Chacón et al., 2007). Muestra de ello es la evolución de Arona y Adeje desde una agricultura marginal de secano y cultivos bajo plástico a una explotación de su frente de mar por una industria de ocio y recreación ligada al turismo de sol y playa (figura 2).

Estas localidades suman a los efectos negativos de una utilización social creciente de su costa los riesgos que pueda ocasionar la escorrentía, cuando se produce. Por ellas discurre el tramo final de los barrancos de 16 cuencas-vertientes alargadas, que la erosión ha abierto en los mantos piroclásticos y coladas fonolíticas, traquíticas y basálticas procedentes de las erupciones acontecidas en el dorso meridional de la Caldera de Las Cañadas del Teide en los dos últimos millones de años (Kereszturi et al., 2013). Constituyen en su conjunto cuencas de drenaje de orden 2 y 3 (63\%) con una extensión media de 0,65 a 3,5 $\mathrm{km}^{2}$, seguidas a cierta distancia por otras de rango 4 y 5 (25\%) de entre 4,4 y $35 \mathrm{~km}^{2}$ de superficie. Unas y otras son surcadas por cauces secos de funcionamiento ocasional y acusada torrencialidad, en respuesta a la aridez climática imperante (Díaz-Pacheco et al., 2019), ya que la precipitación media anual es de $145 \mathrm{~mm}$ en el observatorio de Adeje, aunque se han registrado eventos extremos de más de 158 mm en 24 h (AEMET, 2018). En este contexto, es muy relevante la capacidad de respuesta de tales cauces a unas lluvias que, por su gran concentración espacial y temporal, pueden causar inundaciones en el curso bajo y desembocadura de muchos barrancos, coincidiendo con el emplazamiento de la mayoría de las infraestructuras y de la urbanización.

El riesgo de avenidas está ligado en este territorio a una rápida expansión urbana propiciada por al auge de la actividad turística, con un ritmo constructivo más o menos constante desde la década de 1970. En este marco, la urbanización progresa espacialmente de sureste a noroeste y desde el litoral hacia el interior, pues las edificaciones más antiguas corresponden al núcleo de Los Cristianos, en Arona, mientras las intervenciones más recientes se desarrollan desde Costa Adeje hacia La Caleta, en Adeje (figura 3).

La ocupación se inicia en las áreas de menor pendiente, siendo estas las más próximas a la costa (puerto pesquero de Los Cristianos, en Arona, y Las Américas, sector bajo de San Eugenio y la Caleta, en Adeje). Se caracterizan por una ocupación carente, con frecuencia, de planificación integral desde el comienzo de la implantación de la actividad turística. De ahí que no se hayan tenido en cuenta el trazado de los cursos de agua, dado el carácter discontinuo de la escorrentía, y la ubicación de las superficies de drenaje y desembocadura de los barrancos que aquí finalizan. Conforme se colmata el frente marítimo y sus aledaños, la edificación avanza hacia ámbitos de pendiente más pronunciada (Veriles de Los Tableros y Somada de Los Eres, en Arona, y Fañabé, Torviscas, Miraverde y Risco de Adeje, en Adeje) (figura 3). Se asiste así a un incremento de las coberturas urbanas, con la impermeabilización consiguiente de terrenos surcados por cauces que circulan por suelos privados de su capacidad de infiltración. A esta pérdida acompañan, en la actualidad, la canalización del curso medio de numerosos barrancos, sobre todo cuando discurren bajo la trama urbana, la disposición de viales en sentido transversal a los cauces, la existencia de desagües sin dimensionamiento adecuado y de sistemas deficientes 
de canalización de pluviales y la construcción de paseos marítimos en cotas superiores a la rasante de la desembocadura de los cursos de agua.

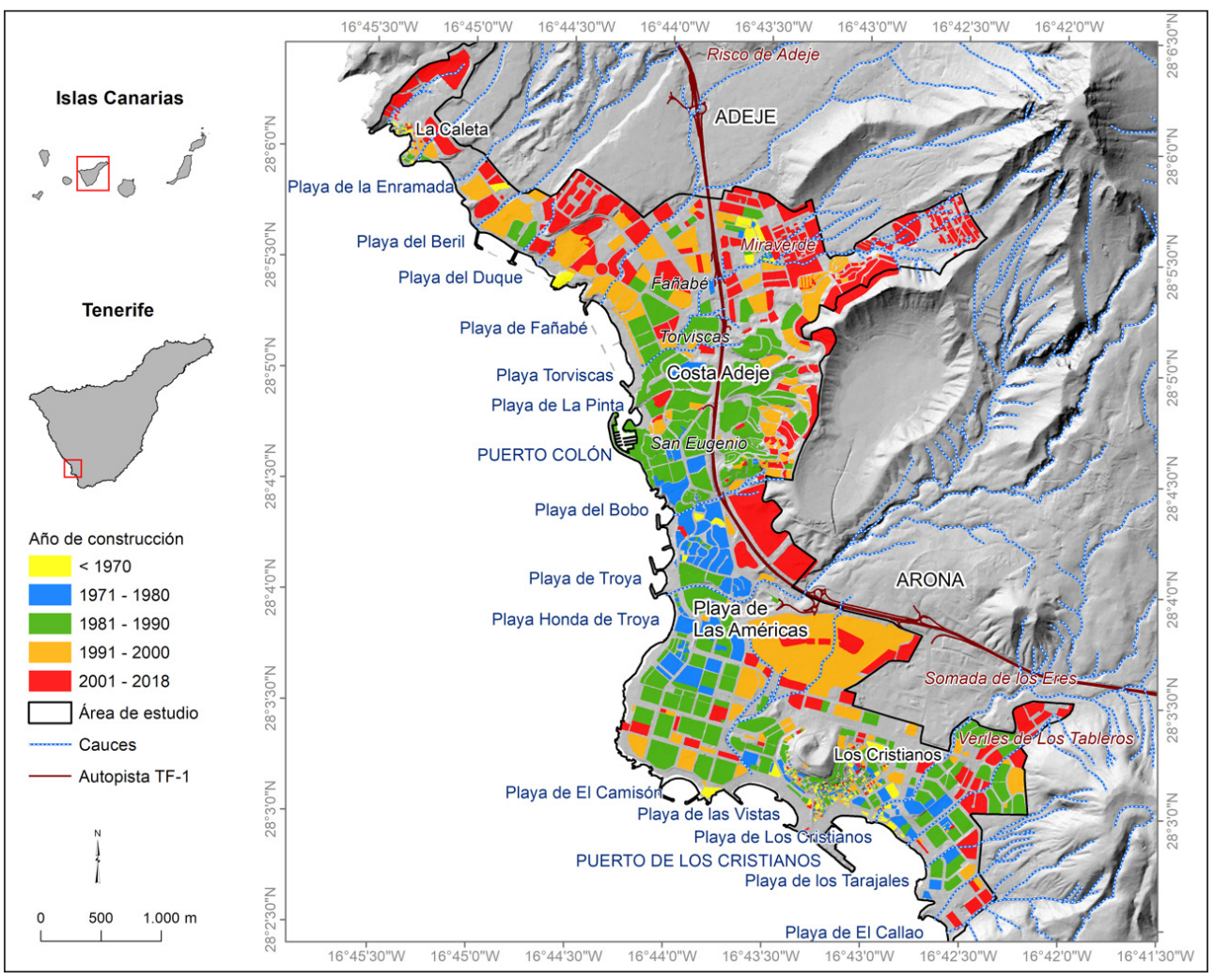

Figura 3. Antigüedad de las edificaciones en la costa de Arona y Adeje (SO de Tenerife). Fuente: Catastro, Grafcan, y PNOA (2019).

La vulnerabilidad ante la amenaza de inundación caracteriza, por todo ello, al área de estudio, aunque con especial relevancia en áreas urbanas con cierto desnivel y capacidad de infiltración reducida. Es aquí donde la circulación del agua en episodios de avenidas súbitas suele producir la interrupción de la movilidad peatonal y del tráfico y el encharcamiento local y puntual de espacios impermeables donde aquella es incapaz de ser evacuada por el drenaje urbano, como locales comerciales y de servicios edificados bajo rasante; destacables son, en este sentido, los almacenes y garajes de infraestructuras hoteleras. Las playas sufren también los efectos de las lluvias intensas en relación con los desbordamientos de cauces, como ha sucedido en algunos de los 21 episodios de precipitación extrema registrados entre 1980 y 2018 (13/12/2002, 18/04/2011) (Díaz-Pacheco et al., 2019). Muestra de tal vulnerabilidad son 
los más de dos millones de euros que el Consorcio de Compensación de Seguros (CCS) pagó entre 1996 y 2016 en concepto de indemnización, por los daños y efectos sobre todo económicos de las avenidas.

\section{Fuentes y MÉTODO}

\section{Fuentes para el análisis de la vulnerabilidad}

La identificación de las áreas expuestas al peligro de avenida y/o inundación (amenaza-exposición) se realiza a través del uso de tres fuentes específicas: de un lado, la Cartografía Nacional de Zonas Inundables a escala 1:25000 (SNCZI, 2014), cuyo establecimiento y administración corresponden al Ministerio de Transición Ecológica (MITECO) de acuerdo con la directiva 2007/60 sobre evaluación y gestión de riesgo de inundación; de otro, el Plan de Defensa de Avenidas (PDA, 2015) dependiente del Consejo Insular de Aguas de Tenerife, que identifica ciertos tramos del cauce de barrancos y una serie de puntos y áreas del territorio insular proclives a experimentar fenómenos de inundación. Y, por último, la prensa local (El Día, Diario de Avisos, La Opinión y Gaceta de Canarias) examinada entre 1970 y 2018, con el fin de obtener evidencias acerca de las consecuencias del impacto y la localización de las inundaciones durante el periodo señalado.

Además de esta información contenida en documentos de planificación para la gestión del riesgo de inundación y el análisis de la prensa, se identificaron episodios de precipitación extrema a través del análisis de los registros de tres estaciones meteorológicas de la isla de Tenerife situadas en el entorno del área de estudio y pertenecientes a AEMET. En la tabla 1 se muestran las series usadas, su localización y los períodos en que se inician y finalizan, con indicación del número de años completos de cada una de ellas.

Tabla 1. Localización, altitud y período temporal de las series pluviométricas utilizadas. Fuente: AEMET (2019).

\begin{tabular}{|c|c|c|c|c|c|c|c|}
\hline \multicolumn{1}{|c|}{ Estación } & Isla & Latitud N & Longitud W & Altitud (m) & Inicio & Fin & Años serie \\
\hline C429I-Tfe. Sur & Tenerife & $27^{\circ} 59^{\prime} 29^{\prime \prime}$ & $16^{\circ} 41^{\prime} 10^{\prime \prime}$ & 12 & $07 / 1980$ & $12 / 2018$ & 37 \\
\hline C418U-Adeje & Tenerife & $28^{\circ} 06^{\prime} 40^{\prime \prime}$ & $16^{\circ} 42^{\prime} 59^{\prime \prime}$ & 266 & $04 / 1944$ & $08 / 2007$ & 25 \\
\hline C429A-Arona & Tenerife & $27^{\circ} 59^{\prime} 29^{\prime \prime}$ & $16^{\circ} 41^{\prime} 10^{\prime \prime}$ & 12 & $01 / 1945$ & $07 / 1994$ & 44 \\
\hline
\end{tabular}

Para realizar la evaluación de la vulnerabilidad se recurre a fuentes que permiten localizar y definir usos, edificaciones y distintas actividades, aunque la unidad mínima de análisis empleada es la parcela catastral. En este contexto, la parcela catastral, establecida en el sistema de gestión de la Dirección General del Catastro del Ministerio de Hacienda de España, adquiere gran relevancia como unidad de análisis. Su empleo en este trabajo obedece a que su demarcación es objetiva, como exactos son sus límites y la naturaleza de su información. Cabe destacar la proporcionada acerca del inmueble o 
conjunto de inmuebles que la ocupa y la relativa, entre otros aspectos, a la superficie total construida, a la parte de esta que se encuentra bajo la rasante y a la antigüedad de la edificación; de igual manera, la relativa a reseñas sobre los usos del suelo susceptibles de extrapolación, que, a pesar de su más difícil acceso, permiten la realización de cálculos para la estimación de la vulnerabilidad (Camarasa et al., 2008). De esta forma, el uso de la parcela catastral facilita medir su pendiente topográfica media, agregar ubicaciones puntuales aprovechables para locales de servicios, comercios, restauración, entre otras construcciones, o desagregar, por extrapolación, datos de población o plazas turísticas. Macías González (2017) la utiliza, mediante ciertas adaptaciones, para la estimación local del riesgo por movimientos de ladera en áreas turísticas costeras del sur de Gran Canaria y para el examen de la impermeabilización del sustrato. A la parcela catastral se añade en el estudio que ahora se realiza, el área ocupada por las playas, donde generalmente se localiza la desembocadura de diversos barrancos.

Otras fuentes consultadas para evaluar la vulnerabilidad son la base de datos geográficos de actividades del Cabildo de Tenerife (2018), de la que procede la información relativa a tipo y ubicación de edificaciones, así como los datos de población residente por núcleos de población proporcionados por el ISTAC (2017).

\section{Método}

En los procesos de evaluación del riesgo, existen múltiples metodologías para calcular la vulnerabilidad (Balica et al., 2012; Birkman, 2007; Cardona, 2005; D'Ercole y Metzger, 2004; Polsky et al., 2003; Rhased y Weeks, 2003; Villagrán de León, 2006). Cuando se plantean a escala local, se suelen centrar en la identificación de diferencias espaciales en el ámbito de una región concreta, para diseñar con posterioridad medidas de reducción del riesgo. Experiencias como las de Aroca-Jiménez et al. (2018) en Castilla-La Mancha se caracterizan por su enfoque estadístico y consideración de la administración municipal como unidad mínima de análisis. A este nivel, la disponibilidad de información estadística y una cierta heterogeneidad ambiental y socio-económica entre los 919 municipios de esa Comunidad Autónoma posibilitan el reconocimiento de desigualdades geográficas. Este hecho es extensivo al análisis en grandes cuencas, como pueden ser los estudios realizados al respecto en América Central (Jiménez et al., 2004). A partir de ellos es factible distinguir con mayor claridad diferentes tipos o ejes de vulnerabilidad - desde la económica y política hasta la social - cuya consideración posibilita el establecimiento de un índice de conjunto.

La cuestión entraña mayor dificultad si el espacio de estudio se reduce a un enclave urbano local con cierta homogeneidad, en cuanto a población residente, número de turistas, densidad urbana, actividad económica y aspectos sociales. Así sucede en el ámbito conformado por Arona y Adeje, donde la distinción del grado de vulnerabilidad puede radicar sólo en matices. En este sentido, la antigüedad de las edificaciones, la identificación de áreas afectadas de manera recurrente por las avenidas, la pendiente del terreno valorada por su importancia en la capacidad de arrastre y erosión de la escorrentía superficial, las construcciones bajo rasante o el aforo pueden tenerse como indicadores, que influyan en la diferenciación espacial de la susceptibilidad de cada una de las diversas estructuras existentes a ser 
dañadas con motivo de un evento de inundación. Examinar la vulnerabilidad a este nivel de detalle suele conllevar, por lo general, importantes esfuerzos a la hora de generar información, en especial si los datos no están siempre disponibles y su obtención requiere gran inversión de tiempo y recursos. Por ello, contar con un procedimiento para su evaluación de forma útil y factible, según los registros con que se puede trabajar y hacerlo a escala local, es de interés notable desde la óptica del planeamiento, sobre todo para la reducción del riesgo.

\section{Cálculo de la vulnerabilidad específica (Vt)}

Durante el Decenio Internacional de los Desastres Naturales declarado por Naciones Unidas en 1990, esta organización publicó un manual dirigido a gestores de planificación para apoyar su labor en la mitigación de desastres naturales. En él se recoge una metodología para la evaluación del riesgo (UNDRO, 1991, p. 71), que, en lo esencial, se sigue empleando en la actualidad. De ahí que constituya la base de muchos de los métodos aquí consultados.

De la consideración de tal metodología resulta que para el cómputo del riesgo total (Rt) es preciso que el mismo sea igual al producto de los elementos en riesgo o vulnerables (E) y el riesgo específico (Rs). Este último se expresa, a su vez, en función del producto entre la amenaza (A) y la vulnerabilidad (V). De este modo:

$$
\mathrm{Rt}=\sum(\mathrm{E})(\mathrm{Rs})=(\mathrm{E})(\mathrm{A} * \mathrm{~V})
$$

El significado otorgado al riesgo específico en este planteamiento guarda gran similitud con la forma en que, por lo general, se concibe el concepto de vulnerabilidad. Lo que en este caso se denomina riesgo específico (Rs) puede ser considerado, entonces, como un cómputo de vulnerabilidad específica $(\mathrm{Vt})$, cuyo cálculo tiene lugar en función del tipo de elemento en riesgo (E). De este modo:

$$
\mathrm{Vt}=(\mathrm{E})(\mathrm{A} * \mathrm{~V})
$$

Donde la vulnerabilidad específica $(\mathrm{Vt})$ está calculada para cada unidad de análisis o parcela catastral. (E) es el factor de ponderación aplicado a los elementos vulnerables de cada parcela catastral de acuerdo a su clasificación según su uso. (A) es la amenaza-exposición calculada en función de los peligros identificados y propiedades físicas (Ej: pendiente del terreno) que afectan a cada unidad de análisis. (V) es un cómputo entre algunos factores socio-económicos simples de la unidad de análisis relacionados con su vulnerabilidad ante avenidas y/o avenidas.

\section{Clasificación y ponderación de los elementos vulnerables (E)}

Los elementos vulnerables (E) se clasifican de acuerdo a sus características frente a la amenaza-exposición (A), lo que se incorpora como un factor de sensibilidad. Este funciona como un valor de ponderación y también como propiedad a partir de la cual la amenaza-exposición (A) y la vulnerabilidad $(\mathrm{V})$ integran unos u otros indicadores. 
Para posibilitar la ponderación de los elementos vulnerables (E), con respecto al contexto donde se acomete el proceso de evaluación, se procede a su agrupamiento según aconseja la metodología de UNDRO (1991). De acuerdo a sus rasgos, la sistematización que aquí se propone parte de una simplificación de la dinámica socio-económica principal en cada parcela. De este modo, se establece un factor de ponderación de los usos de suelo entre 0 y 1 , definido por cómo un episodio específico de inundación puede afectar a los diferentes espacios. Esto hace que usos vinculados, por ejemplo, a las edificaciones turísticas y residenciales presenten un valor de ponderación de 0,75 y 0,5, respectivamente, mayor al de usos como playas $(0,25)$ o aparcamientos $(0,25)$, donde los daños y efectos tanto económicos como sociales van a ser a priori menores.

En este sentido, aunque los usos de suelo son un factor determinante, es posible que la existencia de ciertas actividades influya también en la categorización que se establece. Así, por ejemplo, la presencia de un centro hospitalario en una parcela con edificación residencial puede hacer que se catalogue en función de dicho centro, al ser el agente esencial de la actividad que en ella tiene lugar. Los valores de ponderación establecidos se muestran en la tabla 2.

\section{Identificación de la amenaza-exposición (A)}

Las fuentes citadas posibilitan la localización de las áreas y puntos que, según diversos estudios sobre el desarrollo de distintos planes preventivos y de gestión (SNCZI, 2014; PDA, 2015), se encuentran en riesgo de sufrir inundaciones y avenidas. Riesgo en función bien de la existencia de daños ocurridos en eventos de avenida y/o inundación registrados, o bien por la realización de modelos hidráulicos, que señalan zonas potencialmente inundables para periodos de retorno de 100 y 500 años (SNCZI).

La identificación de los episodios extremos se lleva a cabo mediante el análisis de las series temporales de precipitación diaria, procedentes de las estaciones meteorológicas seleccionadas empleando el Percentil, $\mathrm{P}_{99}$. Tras su individualización se determinan las consecuencias que han podido causar las lluvias, al tiempo que se relacionan con trabajos de riesgo que, en un primer momento, se han desarrollado en el área de estudio (López et al., 2019). En tal sentido, se tienen en cuenta procesos de encharcamiento de áreas específicas, inundación temporal de calles, inundación de edificios de uso residencial, turístico o comercial, colapso puntual y localizado del sistema urbano de evacuación de aguas, afecciones a playas o zonas de baño, etc.

Una vez reconocida y localizada la amenaza se realiza la valoración de la exposición, la cual se aplica a los elementos vulnerables (en riesgo). Cada evidencia de amenaza se contabiliza con el valor de 1, añadiéndose una unidad cada vez que existe coincidencia espacial. De esta manera, si una parcela se encuentra afectada por un área de peligro señalada por el PDA y también por un área de riesgo potencialmente significativa, adquiere una valoración de 2 unidades. Se tiene en cuenta, además, otro factor inherente a las propiedades del terreno donde se extiende la unidad de análisis, como es la pendiente, que también se contabiliza por su estrecha relación con los procesos de escorrentía y flujo laminar de agua y acarreos. En este caso se realiza una clasificación de los valores por cuartil, normalizando de 0 a 1 la valoración y añadiéndola así mismo al cálculo realizado. 


\section{Valoración de la vulnerabilidad}

La vulnerabilidad para cada elemento en riesgo se estima a través de la observación de diferentes indicadores. Su cómputo individual se realiza mediante la agrupación de cada variable en cuartiles para, a continuación, normalizar el valor de 0 a 1 . Con posterioridad, cada uno de estos cómputos se suma para obtener el valor (V) dentro de la ecuación de cálculo de la vulnerabilidad total (Vt). De este modo se identifican: (1) componentes constructivos (Dwyer et al., 2004), como la edad de los inmuebles y la ubicación de edificaciones bajo el nivel de la calzada; (2) el número de comercios, restaurantes y servicios ubicados en cada parcela (Cabildo de Tenerife), que suelen estar generalmente en la primera planta; (3) la superficie de las edificaciones ubicadas bajo rasante; y (4) elementos cuantitativos referidos a las personas, como el aforo de determinadas instalaciones, el número de plazas alojativas o la población residente total desagregada en parcelas, a partir de los registros de 10 núcleos de población que conforman el área de estudio.

Tabla 2. Identificación y valoración de indicadores de vulnerabilidad propuestos. Fuente: Elaboración propia.

\begin{tabular}{|c|c|c|c|c|}
\hline $\begin{array}{c}\text { Agrupación de Elementos } \\
\text { Vulnerables }\end{array}$ & $\begin{array}{l}\text { Indicadores } \\
\text { de Amenaza- } \\
\text { Exposición }\end{array}$ & $\begin{array}{c}\text { Valores } \\
\text { asignados }\end{array}$ & $\begin{array}{l}\text { Indicadores de } \\
\text { Vulnerabilidad }\end{array}$ & Valores asignados \\
\hline \multirow{3}{*}{$\begin{array}{l}\text { 1. Playas y zonas de baño } \\
\text { Factor de ponderación } 0,25\end{array}$} & $\begin{array}{l}\text { Daños consta- } \\
\text { tados (PDA, } \\
\text { prensa) }\end{array}$ & $\begin{array}{l}0 \text { no daño } \\
1 \mathrm{x} \\
\text { evento }\end{array}$ & $\begin{array}{l}\text { Capacidad de } \\
\text { acogida }\end{array}$ & $\begin{array}{l}\text { Asignación según } \\
\text { capacidad de carga y } \\
\text { superficie }\end{array}$ \\
\hline & $\begin{array}{l}\text { Se encuentra en } \\
\text { zona inundable } \\
\text { (SNCZI) }\end{array}$ & $\begin{array}{l}0 \text { no daño } \\
1 \mathrm{x} \\
\text { evento }\end{array}$ & & \\
\hline & $\begin{array}{l}\text { Es desembocadu- } \\
\text { ra de cuenca }\end{array}$ & $\begin{array}{l}0 \text { no daño } \\
1 \mathrm{x} \\
\text { evento }\end{array}$ & & \\
\hline \multirow{3}{*}{$\begin{array}{l}\text { 2. Instalaciones portuarias } \\
\text { Factor de ponderación } 0,25\end{array}$} & $\begin{array}{l}\text { Daños consta- } \\
\text { tados (PDA, } \\
\text { prensa) }\end{array}$ & $\begin{array}{l}0 \text { no daño } \\
1 \mathrm{x} \\
\text { evento }\end{array}$ & $\begin{array}{l}\mathrm{N}^{o} \text { de comer- } \\
\text { cios, equipa- } \\
\text { mientos }\end{array}$ & $\begin{array}{l}\text { Asignación según } \\
\text { número total de } \\
\text { servicios }\end{array}$ \\
\hline & $\begin{array}{l}\text { Se encuentra en } \\
\text { zona inundable } \\
\text { (SNCZI) }\end{array}$ & $\begin{array}{l}0 \text { no daño } \\
1 \mathrm{x} \\
\text { evento }\end{array}$ & & \\
\hline & $\begin{array}{l}\text { Es desembocadu- } \\
\text { ra de cuenca }\end{array}$ & $\begin{array}{l}0 \text { no daño } \\
1 \mathrm{x} \\
\text { evento }\end{array}$ & & \\
\hline
\end{tabular}




\begin{tabular}{|c|c|c|c|c|}
\hline $\begin{array}{l}\text { Agrupación de Elementos } \\
\text { Vulnerables }\end{array}$ & $\begin{array}{l}\text { Indicadores } \\
\text { de Amenaza- } \\
\text { Exposición }\end{array}$ & $\begin{array}{l}\text { Valores } \\
\text { asignados }\end{array}$ & $\begin{array}{l}\text { Indicadores de } \\
\text { Vulnerabilidad }\end{array}$ & Valores asignados \\
\hline \multirow{4}{*}{$\begin{array}{l}\text { 3. Espacios abiertos: Parques, } \\
\text { plazas; parques temáticos; campos } \\
\text { de golf; instalaciones deportivas } \\
\text { Factor de ponderación } 0,25\end{array}$} & $\begin{array}{l}\text { Daños consta- } \\
\text { tados (PDA, } \\
\text { prensa) }\end{array}$ & $\begin{array}{l}0 \text { no daño } \\
1 \mathrm{x} \\
\text { evento }\end{array}$ & Aforo & $\begin{array}{l}\text { Asignación según } \\
\text { superficie }\end{array}$ \\
\hline & $\begin{array}{l}\text { Se encuentra en } \\
\text { zona inundable } \\
\text { (SNCZI) }\end{array}$ & $\begin{array}{l}0 \text { no daño } \\
1 \mathrm{x} \\
\text { evento }\end{array}$ & & \\
\hline & $\begin{array}{l}\text { Es cruzada por } \\
\text { cauce }\end{array}$ & $\begin{array}{l}0 \text { no daño } \\
1 \mathrm{x} \\
\text { evento }\end{array}$ & & \\
\hline & Pendiente media & $\begin{array}{l}<10^{\circ}=0, \\
10^{\circ}- \\
15^{\circ}=0,25 \\
15^{\circ}- \\
30^{\circ}=0,50 \\
30^{\circ}- \\
45^{\circ}=0,75 \\
>45^{\circ}=1\end{array}$ & & \\
\hline \multirow[t]{2}{*}{$\begin{array}{l}\text { 4. Edificaciones residenciales } \\
\text { Factor de ponderación 0,50 }\end{array}$} & $\begin{array}{l}\text { Daños consta- } \\
\text { tados (PDA, } \\
\text { prensa) }\end{array}$ & $\begin{array}{l}0 \text { no daño } \\
1 \mathrm{x} \\
\text { evento }\end{array}$ & $\begin{array}{l}\text { Superficie bajo } \\
\text { rasante }\end{array}$ & $\begin{array}{l}0 \mathrm{~m}^{2}=0 \\
<=50 \mathrm{~m}^{2}=0,25 \\
<=150 \mathrm{~m}^{2}=0,50 \\
<=1200 \mathrm{~m}^{2}=0,75 \\
>1200 \mathrm{~m}^{2}=1\end{array}$ \\
\hline & $\begin{array}{l}\text { Se encuentra en } \\
\text { zona inundable } \\
\text { (SNCZI) }\end{array}$ & $\begin{array}{l}0 \text { no daño } \\
1 \mathrm{x} \\
\text { evento }\end{array}$ & $\begin{array}{l}\mathrm{N}^{\circ} \text { de comer- } \\
\text { cios, equipa- } \\
\text { mientos }\end{array}$ & $\begin{array}{l}\text { Asignación según } \\
\text { número total de } \\
\text { servicios }\end{array}$ \\
\hline \multirow{3}{*}{$\begin{array}{l}\text { 5. Edificios de alojamiento turís- } \\
\text { tico } \\
\text { Factor de ponderación } 0,75\end{array}$} & $\begin{array}{l}\text { Daños consta- } \\
\text { tados (PDA, } \\
\text { prensa) }\end{array}$ & $\begin{array}{l}0 \text { no daño } \\
1 \mathrm{x} \\
\text { evento }\end{array}$ & $\begin{array}{l}\text { Superficie bajo } \\
\text { rasante }\end{array}$ & $\begin{array}{l}0 \mathrm{~m}^{2}=0 \\
<=50 \mathrm{~m}^{2}=0,25 \\
<=150 \mathrm{~m}^{2}=0,50 \\
<=1200 \mathrm{~m}^{2}=0,75 \\
>1200 \mathrm{~m}^{2}=1\end{array}$ \\
\hline & $\begin{array}{l}\text { Se encuentra en } \\
\text { zona inundable } \\
\text { (SNCZI) }\end{array}$ & $\begin{array}{l}0 \text { no daño } \\
1 \mathrm{x} \\
\text { evento }\end{array}$ & $\begin{array}{l}\mathrm{N}^{\circ} \text { de comer- } \\
\text { cios, equipa- } \\
\text { mientos }\end{array}$ & $\begin{array}{l}\text { Asignación según } \\
\text { número total de } \\
\text { servicios }\end{array}$ \\
\hline & Pendiente media & $\begin{array}{l}<10^{\circ}=0, \\
10^{\circ}- \\
15^{\circ}=0,25 \\
15^{\circ}- \\
30^{\circ}=0,50 \\
30^{\circ}- \\
45^{\circ}=0,75 \\
>45^{\circ}=1\end{array}$ & $\begin{array}{l}\text { Plazas de aloja- } \\
\text { miento }\end{array}$ & $\begin{array}{l}<=200=0,25 \\
<=300=0,50 \\
<=500=0,75 \\
>500=1\end{array}$ \\
\hline
\end{tabular}




\begin{tabular}{|c|c|c|c|c|}
\hline $\begin{array}{l}\text { Agrupación de Elementos } \\
\text { Vulnerables }\end{array}$ & $\begin{array}{l}\text { Indicadores } \\
\text { de Amenaza- } \\
\text { Exposición }\end{array}$ & $\begin{array}{l}\text { Valores } \\
\text { asignados }\end{array}$ & $\begin{array}{l}\text { Indicadores de } \\
\text { Vulnerabilidad }\end{array}$ & Valores asignados \\
\hline \multirow{3}{*}{$\begin{array}{l}\text { 6. Edificaciones comerciales, equi- } \\
\text { pamientos y otras actividades } \\
\text { Factor de ponderación } 0,50\end{array}$} & $\begin{array}{l}\text { Daños consta- } \\
\text { tados (PDA, } \\
\text { prensa) }\end{array}$ & $\begin{array}{l}0 \text { no daño } \\
1 \mathrm{x} \\
\text { evento }\end{array}$ & $\begin{array}{l}\text { Superficie bajo } \\
\text { rasante }\end{array}$ & $\begin{array}{l}0 \mathrm{~m}^{2}=0 \\
<=50 \mathrm{~m}^{2}=0,25 \\
<=150 \mathrm{~m}^{2}=0,50 \\
<=1200 \mathrm{~m}^{2}=0,75 \\
>1200 \mathrm{~m}^{2}=1\end{array}$ \\
\hline & $\begin{array}{l}\text { Se encuentra en } \\
\text { zona inundable } \\
(\mathrm{SNCZI})\end{array}$ & $\begin{array}{l}0 \text { no daño } \\
1 \mathrm{x} \\
\text { evento }\end{array}$ & $\begin{array}{l}\mathrm{N}^{o} \text { de comer- } \\
\text { cios, equipa- } \\
\text { mientos }\end{array}$ & $\begin{array}{l}\text { Asignación según } \\
\text { número total de } \\
\text { servicios }\end{array}$ \\
\hline & Pendiente media & $\begin{array}{l}<10^{\circ}=0, \\
10^{\circ}- \\
15^{\circ}=0,25 \\
15^{\circ} \text { - } \\
30^{\circ}=0,50 \\
30^{\circ} \text { - } \\
45^{\circ}=0,75 \\
>45^{\circ}=1\end{array}$ & & \\
\hline \multirow[t]{2}{*}{$\begin{array}{l}\text { 7. Colegios y centros de formación } \\
\text { Factor de ponderación } 1\end{array}$} & $\begin{array}{l}\text { Daños consta- } \\
\text { tados (PDA, } \\
\text { prensa) }\end{array}$ & $\begin{array}{l}0 \text { no daño } \\
1 \mathrm{x} \\
\text { evento }\end{array}$ & $\begin{array}{l}\text { Superficie bajo } \\
\text { rasante }\end{array}$ & $\begin{array}{l}0 \mathrm{~m}^{2}=0 \\
<=50 \mathrm{~m}^{2}=0,25 \\
<=150 \mathrm{~m}^{2}=0,50 \\
<=1200 \mathrm{~m}^{2}=0,75 \\
>1200 \mathrm{~m}^{2}=1\end{array}$ \\
\hline & $\begin{array}{l}\text { Se encuentra en } \\
\text { zona inundable } \\
(\mathrm{SNCZI})\end{array}$ & $\begin{array}{l}0 \text { no daño } \\
1 \mathrm{x} \\
\text { evento }\end{array}$ & $\begin{array}{l}\text { Número de } \\
\text { usuarios }\end{array}$ & $\begin{array}{l}\text { Asignación según } \\
\text { número total de } \\
\text { usuarios }\end{array}$ \\
\hline \multirow{2}{*}{$\begin{array}{l}\text { 8. Hospitales y centros de día y } \\
\text { centros de salud } \\
\text { Factor de ponderación } 1\end{array}$} & $\begin{array}{l}\text { Daños consta- } \\
\text { tados (PDA, } \\
\text { prensa) }\end{array}$ & $\begin{array}{l}0 \text { no daño } \\
1 \mathrm{x} \\
\text { evento }\end{array}$ & $\begin{array}{l}\text { Superficie bajo } \\
\text { rasante }\end{array}$ & $\begin{array}{l}0 \mathrm{~m}^{2}=0 \\
<=50 \mathrm{~m}^{2}=0,25 \\
<=150 \mathrm{~m}^{2}=0,50 \\
<=1200 \mathrm{~m}^{2}=0,75 \\
>1200 \mathrm{~m}^{2}=1\end{array}$ \\
\hline & $\begin{array}{l}\text { Se encuentra en } \\
\text { zona inundable } \\
(\mathrm{SNCZI})\end{array}$ & $\begin{array}{l}0 \text { no daño } \\
1 \mathrm{x} \\
\text { evento }\end{array}$ & $\begin{array}{l}\text { Número de } \\
\text { usuarios }\end{array}$ & $\begin{array}{l}\text { Asignación según } \\
\text { número total de } \\
\text { usuarios }\end{array}$ \\
\hline \multirow{2}{*}{$\begin{array}{l}\text { 9. Servicios de seguridad, emer- } \\
\text { gencias y protección civil } \\
\text { Factor de ponderación } 1\end{array}$} & $\begin{array}{l}\text { Daños consta- } \\
\text { tados (PDA, } \\
\text { prensa) }\end{array}$ & $\begin{array}{l}0 \text { no daño } \\
1 \mathrm{x} \\
\text { evento }\end{array}$ & $\begin{array}{l}\text { Superficie bajo } \\
\text { rasante }\end{array}$ & $\begin{array}{l}0 \mathrm{~m}^{2}=0 \\
<=50 \mathrm{~m}^{2}=0,25 \\
<=150 \mathrm{~m}^{2}=0,50 \\
<=1200 \mathrm{~m}^{2}=0,75 \\
>1200 \mathrm{~m}^{2}=1\end{array}$ \\
\hline & $\begin{array}{l}\text { Se encuentra en } \\
\text { zona inundable } \\
(\mathrm{SNCZI})\end{array}$ & $\begin{array}{l}0 \text { no daño } \\
1 \mathrm{x} \\
\text { evento }\end{array}$ & $\begin{array}{l}\text { Grado impor- } \\
\text { tancia Protec- } \\
\text { ción Civil }\end{array}$ & $\begin{array}{l}\text { Amplifica el factor } \\
\text { de ponderación }\end{array}$ \\
\hline
\end{tabular}




\begin{tabular}{|l|l|l|l|l|}
\hline \multicolumn{1}{|c|}{$\begin{array}{c}\text { Agrupación de Elementos } \\
\text { Vulnerables }\end{array}$} & $\begin{array}{c}\text { Indicadores } \\
\text { de Amenaza- } \\
\text { Exposición }\end{array}$ & $\begin{array}{c}\text { Valores } \\
\text { asignados }\end{array}$ & $\begin{array}{l}\text { Indicadores de } \\
\text { Vulnerabilidad }\end{array}$ & Valores asignados \\
\hline & $\begin{array}{l}\text { Daños consta- } \\
\text { tados (PDA, } \\
\text { prensa) }\end{array}$ & $\begin{array}{l}\text { 0 no daño } \\
\text { evento }\end{array}$ & $\begin{array}{l}\text { Superficie bajo } \\
\text { rasante }\end{array}$ & $\begin{array}{l}\mathrm{O}^{2}=0 \\
<=50 \mathrm{~m}^{2}=0,25 \\
<=150 \mathrm{~m}^{2}=0,50 \\
<=1200 \mathrm{~m}^{2}=0,75 \\
>1200 \mathrm{~m}^{2}=1\end{array}$ \\
\cline { 2 - 5 } $\begin{array}{l}\text { 10. Aparcamientos } \\
\text { Factor de ponderación 0,25 }\end{array}$ & $\begin{array}{l}\text { Se encuentra en } \\
\text { zona inundable } \\
\text { (SNCZI) }\end{array}$ & $\begin{array}{l}\text { 0 no daño } \\
1 \mathrm{x} \\
\text { evento }\end{array}$ & $\begin{array}{l}\text { Capacidad o } \\
\text { aforo }\end{array}$ & $\begin{array}{l}\text { Asignación según } \\
\text { superficie }\end{array}$ \\
\hline
\end{tabular}

Una aplicación práctica del cálculo total de la vulnerabilidad establecido en este trabajo se muestra en el ejemplo recogido en la tabla 3, correspondiente a una parcela con una vocación de uso de alojamiento turístico.

Tabla 3. Cálculo de la vulnerabilidad total (Vt) para una parcela catastral de uso de alojamiento turístico. Fuente: Elaboración propia.

\begin{tabular}{|c|c|c|c|c|c|}
\hline $\begin{array}{c}\text { Elemento } \\
\text { Vulnerable } \\
\text { (E) }\end{array}$ & $\begin{array}{l}\text { Factor de } \\
\text { ponderación } \\
\text { (E) } 0-1\end{array}$ & Amenaza total (A) & $\begin{array}{l}\text { Amenaza } \\
\text { ponderada } \\
\text { (A) } 0-1\end{array}$ & Vulnerabilidad total $(\mathrm{V})$ & $\begin{array}{c}\text { Vulnerabilidad } \\
\text { ponderada }(\mathrm{V}) \\
0-1\end{array}$ \\
\hline $\begin{array}{c}\text { Edificios de } \\
\text { alojamiento } \\
\text { turístico }\end{array}$ & 0,75 & $\begin{array}{c}\text { Afectado por cauce }= \\
+1 \\
\text { Daños Constatado } \\
\text { en Prensa }=+2 \\
\text { Pendiente de } 18^{\circ}= \\
+0,5 \\
\text { TOTAL }=3,5\end{array}$ & 0,33 & $\begin{array}{c}\text { Presenta una superficie } \\
\text { bajo rasante de } 1000 \mathrm{~m}^{2}= \\
+0,75 \\
\text { Presenta } 250 \text { plazas de } \\
\text { garaje }=+0,5 \\
\text { Presenta un total de } 11 \\
\text { comercios }=+0,15 \\
\text { TOTAL }=1,4\end{array}$ & 0,26 \\
\hline
\end{tabular}

En definitiva, para el cálculo de la vulnerabilidad específica $(\mathrm{Vt}=\mathrm{E} * \mathrm{~A} * \mathrm{~V})$ se emplearía el producto del elemento vulnerable por la amenaza ponderada y por la vulnerabilidad ponderada. El resultado final del ejercicio realizado sería de 0,06, valor indicativo de un índice de vulnerabilidad bajo. 


\section{Resultados}

El trabajo realizado permite la identificación de 59 episodios de inundación, de los que 31 causan daños importantes, según información de la prensa. Además, se localizan y cartografían 65 enclaves concretos con problemas de avenida y/o inundación, con diferentes niveles de recurrencia. Sirva aquí el ejemplo del cruce de la autopista (TF1) a la altura de Torviscas, donde la prensa menciona inundaciones e impactos en 5 de los 59 episodios analizados (figura 4).

La aplicación de la metodología diseñada al área de estudio se concreta en un índice con representación cartográfica a escala de parcela catastral. De acuerdo con el difundido método de Jenks (1967), la figura 5 muestra la clasificación del índice de vulnerabilidad en intervalos de ruptura natural. Identificando un total de cinco intervalos en función del nivel de vulnerabilidad, con valores que oscilan entre un mínimo de 0 y un máximo de 0,85. Su representación gráfica se hace mediante los colores empleados por Protección Civil en la confección de los mapas de riesgo, según las escalas estandarizadas a tal efecto. Así, el verde oscuro y el verde claro corresponden a índice muy bajo y bajo, mientras que el amarillo, naranja y rojo son expresión de valores moderado, alto y muy alto, respectivamente.

El resultado más significativo de la utilización del método propuesto es una clara diferenciación espacial del grado de vulnerabilidad. En este sentido, se constata la existencia de un índice muy alto, superior al 0,30 en enclaves puntuales con parcelas coincidentes con áreas inundables (MITECO, figura 4), cuyas avenidas son recogidas en la prensa. Se trata de los ubicados en el interior de Arona, en el entorno de la cuenca hídrica situada entre Fañabé y Torviscas, en Adeje, y en el frente marino y aledaños de las playas de El Bobo y del Beril en ese último municipio. Los núcleos con registros altos $(0,15-0,30)$ son algo más frecuentes. Estos se localizan en las inmediaciones de la playa de Los Cristianos, en Arona pero sobre todo en Puerto Colón y playas de Fañabé, del Duque y La Enramada, en Adeje. La importancia tanto de la amenaza como la exposición es más que evidente en todos estos espacios indudables, aunque no se pueden olvidar los matices que incluye un elemento como la vulnerabilidad en cada uno de los elementos. En relación con ello, es evidente la influencia de la ocupación de laderas de pendiente más o menos pronunciada, de lo que son buena muestra las notables diferencias entre las parcelas urbanas que se asientan ladera arriba de la localidad de San Eugenio y las que lo hacen en la parta más alta de Fañabé. No obstante, aunque la pendiente constituye otro factor más a considerar, se aprecia con claridad que son otros agentes los que determinan en gran medida el valor final del índice, como la tipología de la parcela, el histórico de eventos y el número de servicios. 


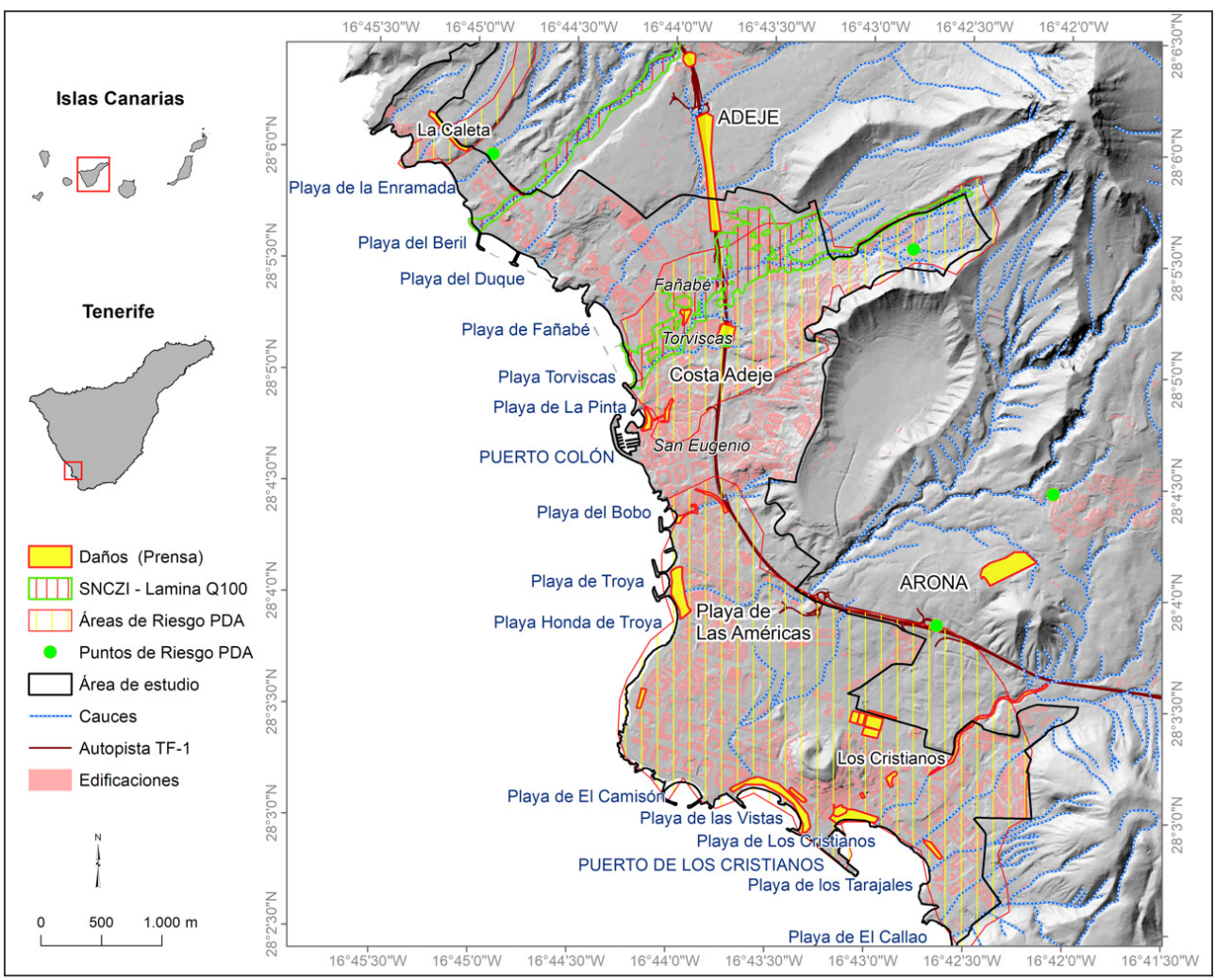

Figura 4. Áreas de amenaza-exposición en la costa de Arona y Adeje (SO de Tenerife). Fuente: PDA (2015), MITECO, Grafcan, IGN; Díaz-Pacheco et al., 2019.

Lo más destacado es, en todo caso, la reducida vulnerabilidad de los sectores en los que se ubican los primeros asentamientos turísticos de Arona y Adeje, como Los Cristianos y Las Américas, respectivamente, aunque es posible que la antigüedad de las edificaciones haya podido condicionar el valor del índice objeto de cálculo. No obstante, son numerosas las gradaciones introducidas por la amenaza-exposición, la pendiente y, sobre todo, por los indicadores de vulnerabilidad. Y ello en un tejido urbano con una densidad edificatoria alta, que crece paralela a la línea de costa mediante la mezcla de tipologías constructivas (Tortosa-Esquembre, 2016), en un proceso de explotación intensiva del suelo falto, al menos en sus inicios, de planificación adecuada (Díaz-Pacheco et al., 2019). La combinación de todos estos factores se concreta en una mayor repercusión económica de la ocupación turística, aforos, etc. 


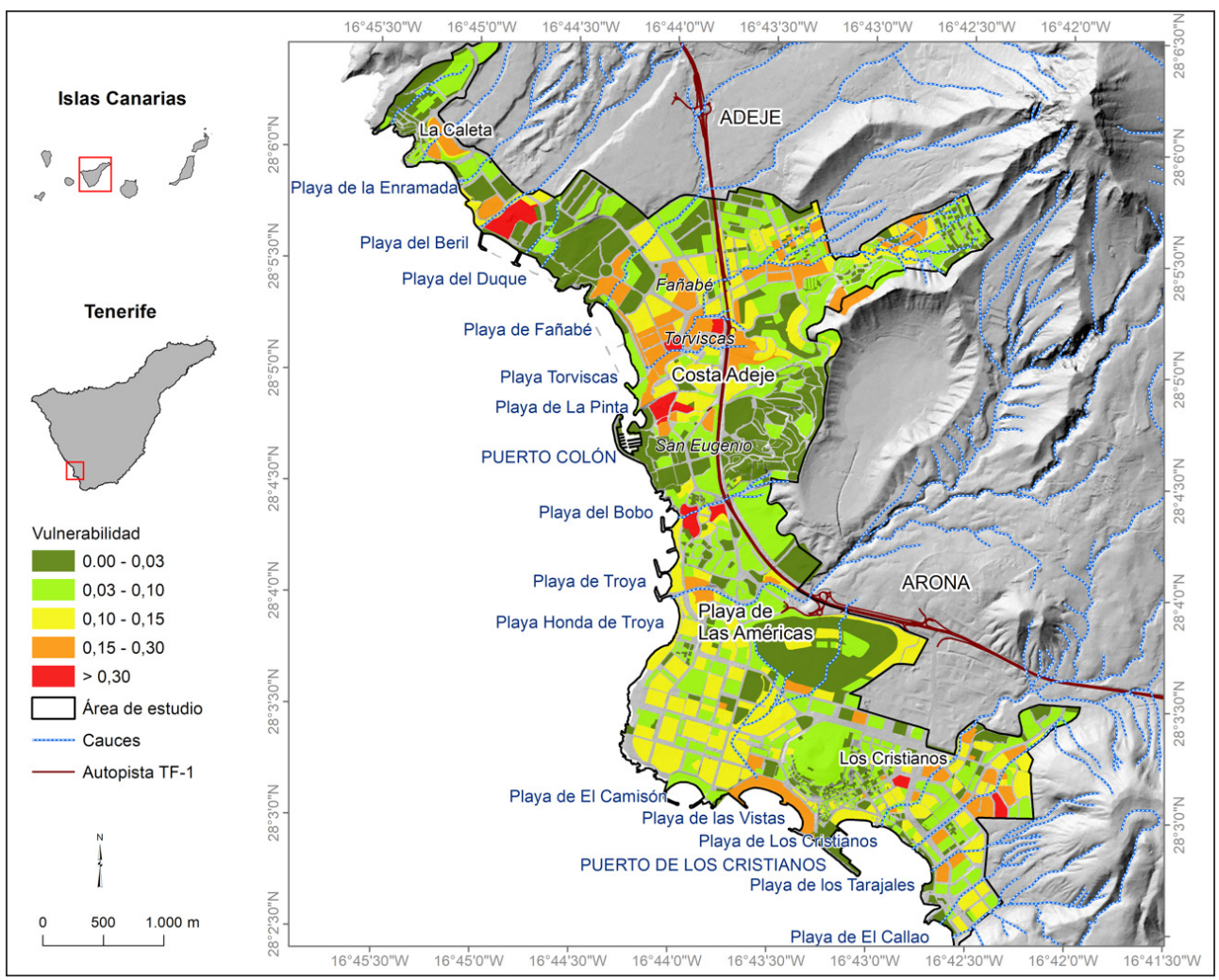

Figura 5. Índice de vulnerabilidad por riesgo de inundación a escala de parcela catastral en la costa de Arona y Adeje (SO de Tenerife). Fuentes: Dirección General del Catastro de SC de Tenerife, Cabildo de Tenerife, PDA (2015), IGN, Grafcan, Díaz-Pacheco et al., 2019.

\section{Conclusiones}

En este trabajo se ha desarrollado un método sencillo de valoración y zonificación de la vulnerabilidad frente al riesgo de inundación en áreas turísticas costeras de espacios insulares, caracterizadas por una precipitación media anual muy baja, pero de intensidad acusada. Este hecho, unido a una urbanización acelerada por la presión turística, incrementa el factor de riesgo de inundación. El peligro en estos enclaves se relaciona con procesos supeditados a la transferencia de caudal en un contexto marcado por la ocupación de pequeños cauces y barranqueras, en los que se ha impuesto una disminución significativa de la capacidad de absorción del sustrato, conforme se ha transformado en pavimento urbano.

El método propuesto posibilita el establecimiento de claras diferencias de vulnerabilidad en unidades catastrales, con un índice que se puede calcular con datos e información disponible de fácil acceso. 
Aunque no se haya efectuado el análisis habitual de las consecuencias y daños relacionados con el calado, así como con otros aspectos de naturaleza económica, los indicadores empleados caracterizan perfectamente la vulnerabilidad en este tipo de enclaves. El índice empleado contribuye, en definitiva, al planteamiento teórico a partir del cual abordar el análisis y diseño de acciones para planificar la evaluación y reducción del riesgo de inundación a escala local. Su interés es manifiesto en ámbitos costeros insulares, muy sensible a los impactos de las actuaciones antrópicas conforme se incrementa su utilización social. Uno espacios que son altamente vulnerables frente a los impactos del cambio climático, tanto por sus rasgos medioambientales como socioeconómicos, más aún cuando como en el caso del presente estudio poseen una alta dependencia de la actividad turística. En este contexto, el establecimiento de metodologías de evaluación de la vulnerabilidad, como la de esta investigación, puede contribuir a definir acciones concretas de adaptación al cambio climático, para evitar así la pérdida de competitividad y aumentar la resiliencia de destinos turísticos maduros, como el suroeste de Tenerife.

\section{Agradecimientos}

Este trabajo se inserta en el proyecto "Análisis del impacto de las inundaciones en áreas turísticas costeras: Canarias, laboratorio natural de resiliencia” (INTUCAN), que financia la Agencia Canaria de Investigación, Innovación y Sociedad de la Información del Gobierno de Canarias y los fondos FEDER.

\section{ReFerencias}

AEMET (2018): Datos del observatorio meteorológico de Arona C427A.

Aroca-Jiménez, E., Bodeque, J., García, J. y Díez-Herrero, A. (2018): A quantitative methodology for the assessment of the regional economic vulnerability to flash floods. Journal of Hidrology, 565, 386-399.

Camarasa Belmonte, A. M., López-García, M. J. y Soriano García, J. (2008): Cartografía de vulnerabilidad frente a inundaciones en llanos mediterráneos: caso de estudio del Barranc de Carraixet y Rambla de Poyo. Serie Geográfica - Profesora María de los Ángeles Díaz Muñoz, In Memoriam, 14, 75-91.

Cardona A. O. (2006): "Midiendo lo Inmedible" Indicadores de Vulnerabilidad y Riesgo. Universidad Nacional de Colombia - Sede Manizales.

Cardona, O.D., Van Aalst, M.K., Birkmann, J., Fordham, M., Mcgregor, G., Perez, R., Pulwarty, R.S., Schipper, E.L.F., \& Sinh, B.T. (2012): A Special Report of Working Groups I and II of the Intergovernmental Panel on Climate Change (IPCC). Cambridge University Press, Cambridge, UK, and New York, NY, USA, 65-108.

Balica, S. F., Wright, N. G. \& Van Der Meulen, F. (2012): A flood vulnerability index for coastal cities and its use in assessing climate change impacts. Natural hazards, 64(1), 73-105. 
Birkmann, J. (2007): Risk and vulnerability indicators at different scales: Applicability, usefulness and policy implications. Environmental hazards, 7(1), 20-31.

Burton, I., Kates, R. \& White, G. (1993): The environment as hazard. UK, Guilford press.

De León, V., y Carlos, J. (2006): Vulnerability: a conceptional and methodological review. Germany, UNU. EHS.

D'Ercole, R. y Metzger, P. (2004): Vulnerabilidad del Distrito Metropolitano de Quito. Chile, AH/Editorial.

Dwyer, A., Zoppou, C., Nielsen, O., Day, S. y Roberts, S. (2004): Quantifying social vulnerability: a methodology for identifying those at risk to natural hazards. Australia, Australian Government.

DÍaz-Pacheco, J., Yanes, A., López-Díez, A., Máyer, P., Dorta, P. (2019): Relación entre episodios de lluvia intensa y daños producidos por inundaciones en áreas turísticas costeras de clima árido: el Sur de Tenerife (1980-2018), En Durán, R., Guillén, J. y Simarro, G. (Eds), X Jornadas de Geomorfología Litoral. Libro de ponencias (pp. 17-30) Castelldefels, (ICM) Libros y partes de libros

Füssel, H.-M. \& Klein, R.J.T. (2006): Climate change vulnerability assessments: an evolution of conceptual thinking. Climatic Change, 75, 301-329.

IPCC, 2014: Climate Change 2014: Impacts, Adaptation, and Vulnerability. Part A: Global and Sectoral Aspects. Contribution of Working Group II to the Fifth Assessment Report of the Intergovernmental Panel on Climate Change [Field, C.B., V.R. Barros, D.J. Dokken, K.J. Mach, M.D. Mastrandrea, T.E. Bilir, M. Chatterjee, K.L. Ebi, Y.O. Estrada, R.C. Genova, B. Girma, E.S. Kissel, A.N. Levy, S. MacCracken, P.R. Mastrandrea, and L.L.White (eds.)]. Cambridge University.

Press, Cambridge, United Kingdom and New York, NY, USA, 1132 ppJenks, G. F. (1967). The data model concept in statistical mapping. International yearbook of cartography, 7, 186-190.

Jiménez, F., Velásquez, S. y Faustino, J. (2004): Análisis integral de la vulnerabilidad a amenazas naturales en cuencas hidrográficas de América Central. VI Semana Científica (Resúmenes). Turrialba, CR, CATIE, 50-53.

Kereszturi, G., Geyer, A., Martí, J., Németh, K. \&Dóniz-Páez, J. (2013): Evaluation of morphometry-based dating of monogenetic volcanoes - a case study from Bandas del Sur, Tenerife (Canary Islands). Bulletin of Volcanology, 75-734: 1-19.

López-Díez, A.; Máyer, P.; Díaz-Pacheco, J.; Dorta, P. (2019): Rainfall and Flooding in Coastal Tourist Areas of the Canary Islands (Spain). Atmosphere, 10, 809.

Macías González, F. (2017): La problemática ambiental de urbanizaciones turísticas costeras: el ejemplo de Puerto Rico-Amadores, Gran Canaria (España). Tesis Doctoral dirigida por Pérez-Chacón, E., Gran Canaria, Universidad de Las Palmas de Gran Canaria, Repositorio (CRIS). < http://hdl.handle. net/10553/54052> (consulta: 20/01/2020).

Mccarthy, J.J., Canziani, O.F., Leary, N.A., Dokken, D.J. \& White, K.S. (2001): Climate Change 2001: Impacts, Adaptation, and Vulnerability. Working Group II of the Intergovernmental Panel on Climate Change, Cambridge University Press, Cambridge, UK.

Mapama (2015): Guía para la Elaboración de Planes Locales de Adaptación al Cambio Climático. Tecnalia. Victoria-Gazteiz. 
O'brien, K., Sygna, L., Leinchenko, R., Adger, W.N., Barnett, J., Mitchell, T., Schipper, L., Tanner, T., Vogel, C. \& Mortreux, C. (2008): Disaster Risk Reduction, Climate Change Adaptation and Human Security. GECHS Report 2008:3, Global Environmental Change and Human Security, Oslo, Norway.

Pérez-Chacón, E. Hernádez-Calvento, L. y Yanes, A. (2007): Transformaciones humanas y sus consecuencias sobre los litorales de las Islas Canarias. En Etienne, S. et Paris, R. (Eds): Les littoraux volcaniques. Une aproche environnementale. Clermont-Ferrand, Press Universitaires Blaise-Pascal, 173-191.

PDA (2015): Plan de Defensa contra Avenidas. Cabildo Insular de Tenerife. CIATF. INCLAN.

Polsky, C., Schröter, D., Patt, A., Gaffin, S., Martello, M.L., Neff, R., Pulsipher, A. \& Selin, H. (2003): Assessing Vulnerabilities to the Effects of Global Change: An Eight-Step Approach. Belfer Center for Science and International Affairs Working Paper, Environment and Natural Resources Program, John F. Kennedy School of Government, Harvard University, Cambridge, Massachusetts.

Rashed, T., \& Weeks, J. (2003): Assessing vulnerability to earthquake hazards through spatial multicriteria analysis of urban areas. International Journal of Geographical Information Science, 17(6), 547-576.

Roig, Fco., Prieto, J. A. M., Perea, A. R., Mir-Gual, M., y Pons, G. X. (2011): La gestión ambiental de los sistemas playa-duna: el caso de las Islas Baleares, En Sanajume, E. y Gracia, F.J. (Eds.): Las dunas en España. Cádiz, Ed. S.E.G. 683-699.

Ruiz Pérez, M. (2011): Vulnerabilidad territorial y evaluación de daños postcatástrofe: una aproximación desde la geografía del riesgo. Tesis Doctoral dirigida por Gutiérrez Puebla, J y Grimalt Gelabert, M. Madrid, Universidad Complutense, Repositorio. <https://eprints.ucm.es/12850/> (consulta: 20/01/2020).

Schröter, D., Polsky, C. \& Patt, A. G. (2005): Assessing vulnerabilities to the effects of global change: an eight step approach. Mitigation and Adaptation Strategies for Global Change, 10(4), 573-595.

Undro (1991): Mitigating natural disasters: Phenomena, effects and options: A manual for policy makers and planners. UN. Office of the Disaster Relief Co-Ordinator. Nueva York, US.

Cómo citar este artículo:

Díaz Pacheco, J., López Díez, A., Yanes Luque, A., Dorta Antequera, P., Máyer Suárez, P. (2020). Propuesta metodológica para estimar la vulnerabilidad local por inundación en áreas turísticas costeras de clima árido: aplicación al litoral de Arona y Adeje (SO de Tenerife). Cuadernos de Geografía, 104, 87-106.

https://doi.org/10.7203/cguv.104.16570

\section{(c) $(1) \Theta \Theta$}

Este obra está bajo una licencia de Creative Commons Reconocimiento-NoComercial-SinObraDerivada 4.0 Internacional. 